Original article

\title{
Histopathological and biochemical evaluation of paeoniflorin administration in an experimental periodontitis model
}

\author{
Çağla Gül Gürkan ${ }^{1)}$, Gonca Çayır Keleș ${ }^{2)}$, Sevda Kurt ${ }^{3)}$, Alper Çiftçi ${ }^{4)}$, Bülent Ayas ${ }^{5)}$, Şevki Güler ${ }^{6}$, \\ and Burcu Özkan Çetinkaya ${ }^{7)}$
}

${ }^{1)}$ Bafra Public Oral Health Center, Samsun, Turkey

${ }^{2)}$ Department of Periodontology, Faculty of Dentistry, İstanbul Okan University, İstanbul, Turkey

3) Department of Periodontology, Faculty of Dentistry, Recep Tayyip Erdoğan University, Rize, Turkey

4) Department of Microbiology, Faculty of Veterinary Medicine, Ondokuz Mayıs University, Samsun, Turkey

5) Department of Histology and Embriology, Faculty of Medicine, Ondokuz Mayıs University, Samsun, Turkey

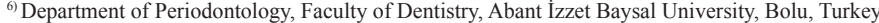

Department of Periodontology, Faculty of Dentistry, Ondokuz Mayıs University, Samsun, Turkey

\begin{abstract}
The purpose of this study was to evaluate the effects of administered Paeoniflorin (Pae) on periodontal tissues within an experimental periodontitis model. Forty male Wistar rats were used in this study and experimental periodontitis was created in all rats except in the control group ( $n=10$, first group). In the periodontitis group, experimental periodontitis was created but no other application was performed ( $n=10$, second group). In the other groups created experimental periodontitis, systemic Pae ( $n=10$, third group) or saline ( $n=10$, fourth group) was applied. A biochemical analysis of the gingival vascular endothelial growth factor (VEGF) levels and a histomorphometric analysis (measurements of the area of alveolar bone, alveolar bone resorption, and attachment loss) were performed. In the Pae group, the area of the alveolar bone was increased, while alveolar bone resorption and attachment loss decreased. Gingival VEGF levels increased in all groups that created experimental periodontitis and the greatest increase seen in the Pae group. Histomorphometric and biochemical analyses in this study suggest that Pae has a curative effect on periodontal tissues. However, additional studies are needed to confirm these results.
\end{abstract}

Keywords; experimental periodontitis, histology, herbal medicine

\section{Introduction}

Periodontitis is a multifactorial, chronic inflammatory disease of the periodontium caused by interactions between periopathogens and the host's defense system, leading to the destruction of tissues around the teeth $[1,2]$. In addition to the direct effects of these pathogenic microorganisms, periodontal destruction occurs from indirect mechanisms related to the host's immune response [3]. Some changes in periodontal vascular structures have been observed during the development of periodontal disease. The various cytokines and growth factors also are effective in regulating angiogenesis, which increases in infected tissues [4-7].

Vascular endothelial growth factor (VEGF), a member of the plateletderived growth factor superfamily, is an important glycoprotein primarily found in endothelial cells. VEGF regulates angiogenesis, increases vascular permeability, stimulates endothelial cell proliferation and differentiation, and induces migration of endothelial cells. This regulation occurs in both physiologic and pathologic events such as wound healing, ischemia, cancer, and inflammation [8-11]. A relationship exists between periodontal disease and VEGF; however, there are conflicting results regarding the role of VEGF in the pathogenesis of periodontal disease [6,7,11,12]. One study noted that VEGF may be associated with both periodontal tissue remodel-

Correspondence to Dr. Sevda Kurt, Department of Periodontology, Faculty of Dentistry, Recep Tayyip Erdoğan University, Rize 53100, Turkey

Fax:+90-464-222-0002 E-mail: dt.sevdakurt@hotmail.com

J-STAGE Advance Publication: October 5, 2019

Color figures can be viewed in the online issue at J-STAGE.

doi.org/10.2334/josnusd.18-0427

DN/JST.JSTAGE/josnusd/18-0427 ing and tissue destruction [7]; however, Çetinkaya et al. (2007) found that VEGF expression was associated with recovery from periodontal disease rather than tissue destruction [12].

Paeoniflorin (Pae) is a monoterpene glycoside isolated from the roots of the Paeoniae lactiflora plant and has been used as a medicinal herb in traditional Chinese medicine [13]. It is a significant bioactive component of the in total glycosides of the extract obtained from the roots and is responsible for their biologic effects [13]. Pae has been shown to have anti-inflammatory and immunoregulatory effects and has been used for many years in the treatment of autoimmune/inflammatory diseases such as rheumatoid arthritis, systemic lupus erythematosus, diabetic nephropathy, and Sjögren syndrome [13-15]. Pae suppresses bone resorption and, by stimulating bone formation, has a positive effect on bone metabolism and remodeling [16,17]. Additionally, Pae has been shown to suppress excessive osteoclastic activity $[16,18]$. Pae has been approved by China's State Food and Drug Administration as a disease-modifying drug in the treatment of rheumatoid arthritis [19]. Given its systemic effects, Pae might be a treatment for metabolic syndromes and systemic diseases associated with bone loss.

The hypothesis of this study was that Pae is effective as a new curative agent in the treatment of periodontal diseases by regulating the host response. Although many studies have evaluated the anti-inflammatory effects of Pae on multiple diseases, there is possibly only one recent study that has examined the effects of Pae on hard and soft tissues in periodontal disease [20]. The present study was based on the hypothesis that Pae has a curative effect on bone attachment and loss. This study was designed to evaluate the anti-inflammatory activity of systemic Pae on periodontal tissue using histomorphometric and biochemical analyses of rats with experimental periodontitis.

\section{Materials and Methods}

\section{Subjects and design}

Forty male Wistar albino rats (weighing 150-250 g) were used in this study. The animals were kept in cages with controlled environmental conditions $\left(22-1{ }^{\circ} \mathrm{C} ; 50 \%\right.$ humidity) with light and dark cycles (12:12 h). They were given food and water ad libitum. All protocols were approved by the Ondokuz Mayis University's Ethical Committee of Animal Research (protocol no: 2014/27).

The subjects were divided randomly into four equal groups. Silk ligatures were tied around the mandibular first molars of all rats except the control group. The ligatures were kept in place for 15 days to create an experimental periodontitis model [11,21].

The groups were treated as follows:

- Group 1: control group (healty and without any treatment) $(n=10)$.

- Group 2: periodontitis group. Sacrification was performed after the creation of experimental periodontitis $(n=10)$.

- Group 3: Pae group. After the formation of experimental periodontitis, ligatures were removed and systemic Pae was applied per os for 7 days. Subsequently, sacrification was performed $(n=10)$. 


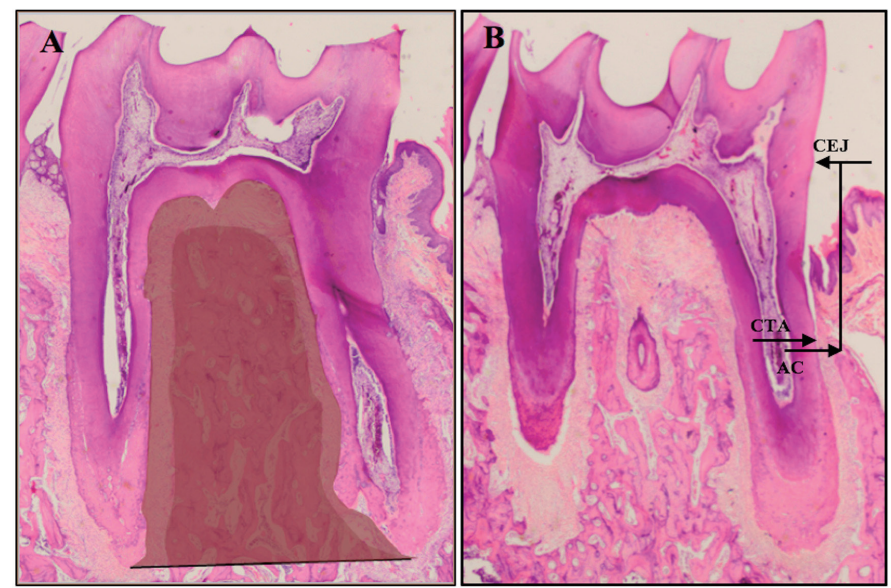

Fig. 1 Reference areas for histomorphometric analysis (Hematoxylin and eosin staining method; magnification, $4 \times$; bar $=100 \mu \mathrm{m}$ ). A; calculation of the alveolar bone area (in the scanned figure, alveolar bone area $=$ dark area, furcation area $=$ dark + light area). $\mathrm{B}$; linear measurement of alveolar bone resorption and attachment loss (CEJ, cemento-enamel junction; CTA, connective tissue attachment; AC, alveolar crest)

- Group 4: saline group. After the formation of experimental periodontitis, ligatures were removed and systemic saline was applied for 7 days $(0.2 \mathrm{~mL})$. Subsequently, sacrification was performed $(n=10)$.

In Pae group, Pae was applied systemically at a dose of $100 \mathrm{mg} / \mathrm{kg}$ dissolved in $0.2 \mathrm{~mL}$ saline solution. This solution was mixed by vortexing and applied by gavage [22]. The saline group was a control group for the Pae group.

\section{Histomorphometric analyses}

Following sacrifice, left mandibular samples were fixed in $10 \%$ neutral formalin and decalcified for 14 days for histomorphometric examination (in $8 \%$ formic acid). Then, the samples were washed for $12 \mathrm{~h}$ and routine histologic evaluation was performed. Sections $5-\mu \mathrm{m}$ thick were taken from the first molar tooth in a mesiodistal direction using a rotary microtome and stained with hematoxylin \& eosin (H\&E). Three different histometric measurements were obtained from each sample in the mesiodistal sections, and the arithmetic mean of the measurements was calculated. In the H\&Estained sections, artificial standard limits were established. Reference volumes of the anatomical structures were identified to determine changes in the periodontal tissues. A light microscope (Olympus BX50 research microscope; Tokyo, Japan) were used for performing histomorphometric analysis and a monitor with a camera apparatus (Olympus DP26 digital camera) were used for transferring the images, the following parameters were evaluated [23]:

1) area of alveolar bone (AA): alveolar bone area / furcation area (ratio, $\%)$;

2) alveolar bone resorpsion (ABR): distance from the cemento-enamel junction to the alveolar bone crest $(\mu \mathrm{m})$;

3) attachment loss (AL): distance between the cemento-enamel junction and the most coronal extent of the connective tissue attachment $(\mu \mathrm{m})$. Designation of the reference areas for histomorphometric analysis is shown in Fig. 1.

\section{Biochemical analysis}

Gingival tissues surrounding the right mandibular first molar teeth were removed using excisional biopsy. The samples were placed into Eppendorf tubes and stored at $-80^{\circ} \mathrm{C}$ until biochemical analysis.

Tissue extractions were performed according to kit procedures. Samples were washed with phosphate buffered saline $(0.01 \mathrm{~mol} / \mathrm{L}, \mathrm{pH}$ 7.0-7.2) to remove debris and then dried. The tissue was weighed before being cut into small pieces with a scalpel. Tissue pieces were placed in a thick glass tube and dissolved with $1 \mathrm{~mL}$ phosphate buffered saline containing protease inhibitor ( $5 \mu \mathrm{g} / \mathrm{mL}$ aprotinin, $1 \mathrm{mM}$ EDTA) to give $10 \mathrm{mg}$ tissue/ $\mathrm{mL}$. The dissolved samples were sonicated five times for $30 \mathrm{~s}$ each time using a UV2200, Bandelin Elc. device. Following sonication, the suspension was centrifuged and the supernatant was removed. The supernatant was stored at $-20^{\circ} \mathrm{C}$ until enzyme-linked immunosorbent assay (ELISA)
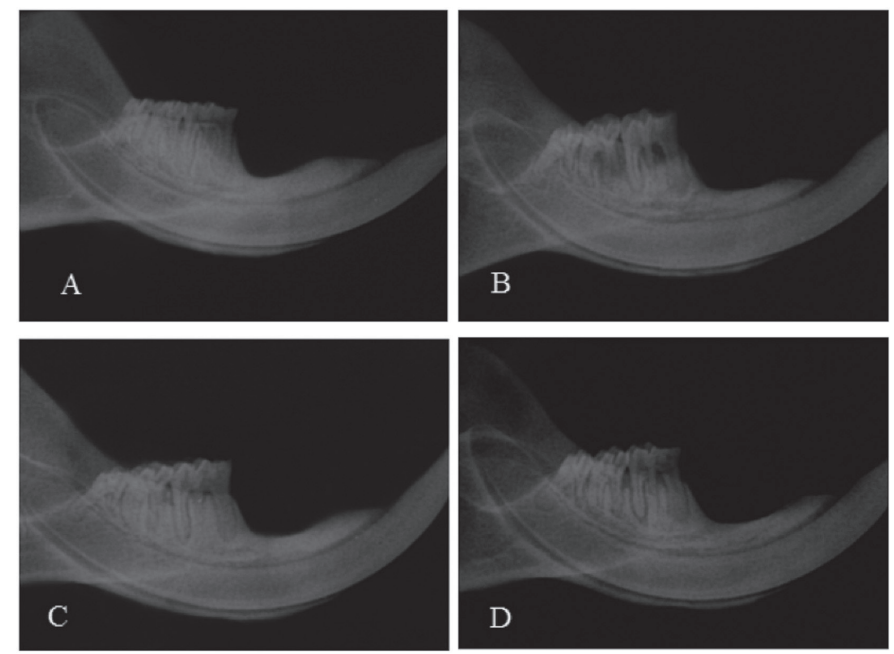

Fig. 2 Radiographic images of the groups. A, control group; B, periodontitis group; C, Pae group; $\mathrm{D}$, saline group

analysis was performed. The VEGF levels of rats' gingival tissue were analyzed in each $150-\mu \mathrm{L}$ sample by ELISA at $450-550 \mathrm{~nm}$ according to the kit manufacturer's protocol (Thermo Fisher Scientific, ERVEGFACL; Schwerte, Germany).

\section{Statistical analysis}

Statistical analysis was performed using SPSS 21.0 Packet Data Program (SPSS 21.0 Software Package Program Inc., Chicago, IL, USA). The data were tested for normality using the Shapiro-Wilk test. All group comparisons were performed using the Kruskal-Wallis test as analysis of the data did not show a normal distribution. Pairwise comparisons of the groups with statistically significant differences were analyzed using a Bonferroniadjusted Mann-Whitney $U$ test. A $P<0.008$ was considered statistically significant in the analysis of the six possible pairwise comparisons.

\section{Results}

\section{Clinical findings}

Pae was tolerated by all animals, and no complications occurred following periodontitis formation and systemic drug administration. By day 15 , significant alveolar bone and AL were detected clinically and radiographically in the groups created experimental periodontitis compared with the control group. Radiographic images of the groups are shown in Fig. 2.

\section{Histomorphometric findings}

AA $(\%)$, ABR $(\mu \mathrm{m})$, and AL $(\mu \mathrm{m})$ values are listed in Table 1. Histomorphometric measurements are found in Fig. 3, and the furcation area of the first molar tooth in the mesiodistal sections are noted in Fig. 4.

AA in the furcation area was significantly greater in the Pae group than in the saline group $(P=0.000)$. There was no statistically significant difference between the saline and periodontitis groups $(P=0.393)$. There was a statistically significant decrease in $\mathrm{AL}$ in the Pae group compared with the periodontitis and saline groups $(P=0.000)$. Among the groups created experimental periodontitis, the $\mathrm{ABR}$ was the lowest in the Pae group $(P=$ $0.004)$.

\section{Biochemical findings}

Table 2 presents gingival VEGF levels of the study groups. Gingival VEGF levels were significantly higher in the groups created experimental periodontitis than in the control group $(P=0.000)$. Gingival VEGF levels were also significantly higher in the Pae group than the periodontitis and saline groups $(P=0.000)$.

\section{Discussion}

This study was performed to research the anti-inflammatory effect of systemic Pae administration on the periodontium of rats with experimental 
Table 1 Area of alveolar bone (\%), alveolar bone resorpsion $(\mu \mathrm{m})$, and attachment loss $(\mu \mathrm{m})$

\begin{tabular}{|c|c|c|c|}
\hline Group & Area of alveolar bone $\mathrm{e}^{\mathrm{a}}$ & Alveolar bone resorpsion ${ }^{a}$ & Attachment loss ${ }^{\mathrm{a}}$ \\
\hline \multirow[t]{2}{*}{1} & $75.18(72.55-77.51)$ & $618.41(544.80-655.00)$ & $287.83(240.66-368.33)$ \\
\hline & $75.07 \pm 1.62$ & $614.13 \pm 35.49$ & $297.56 \pm 40.19$ \\
\hline \multirow[t]{2}{*}{2} & $46.85(40.13-50.70)^{b}$ & $1,411.55(1,302.6-56.33)$ & $1,052.24(1,012.12-1,191.52)$ \\
\hline & $46.22 \pm 3.76$ & $1,417.89 \pm 89.69$ & $1,080.00 \pm 57.88$ \\
\hline \multirow[t]{2}{*}{3} & $68.60(62.00-68.99)$ & $1,048.48(1,005.31-1,168.18)$ & $551.40(402.81-597.40)$ \\
\hline & $66.78 \pm 2.77$ & $1,076.08 \pm 69.23$ & $521.76 \pm 80.16$ \\
\hline \multirow[t]{2}{*}{4} & $49.29(43.88-55.53)^{\mathrm{b}}$ & $1,279.09(1,070.53-1,380.50)$ & 856.57 (770.21-914.67) \\
\hline & $49.50 \pm 5.45$ & $1,249.09 \pm 112.60$ & $848.48 \pm 58.09$ \\
\hline
\end{tabular}

Data are expressed as median, minimum, maximum, and mean \pm standard deviation. ${ }^{a}$ There are statistically significant differences between all groups except the group without differences $(P<0.008)$. ${ }^{b}$ There is no statistically significant difference between the groups $(P>0.008)$. Group 1 , control group; Group 2, periodontitis group; Group 3, Pae group; Group 4, saline group
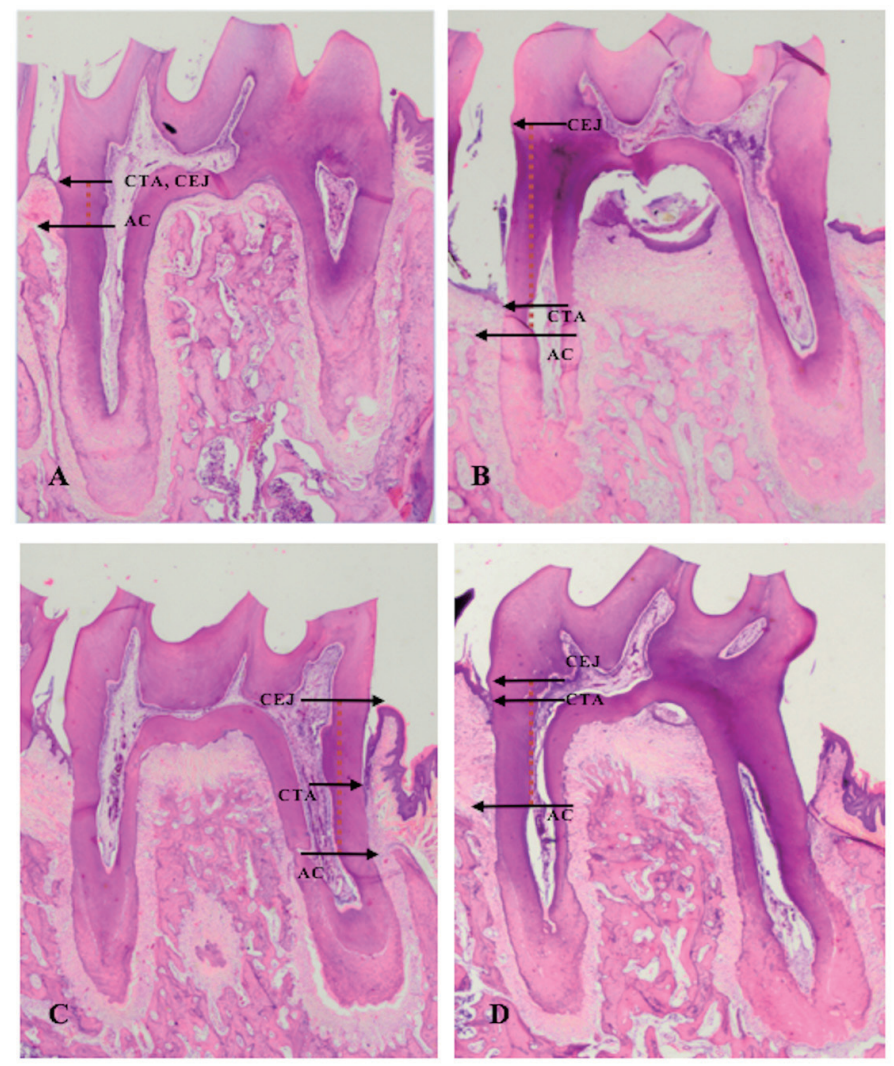

Fig. 3 Sections from the mesio-distal aspects of the mandibular first molars and periodontal tissues (H\&E; magnification, $4 \times$; bar $=100 \mu \mathrm{m}$ ). CTA, connective tissue attachment; CEJ, cemento-enamel junction; AC, alveolar crest. A, control group; B, periodontitis group; C, Pae group; D, saline group

periodontitis using histomorphometric and biochemical analyses.

Although various techniques have been used to generate experimental periodontitis in rats, endotoxin injection and ligation of teeth are the most commonly used techniques [24]. When periodontitis is induced by endotoxin injection, periodontal disease is not induced directly by periodontal pathogens but acute and severe inflammation occurs [24,25]. It has also been noted that additional trauma is present in the molar region following endotoxin injection because it requires continuous injection throughout the experimental period [26]. However, placement of ligatures around the teeth allows plaque build-up causing ulceration of the sulcular epithelium. This study used a experimental periodontitis model generated by ligature, which is a simple and reliable technique for evaluating the progression of periodontal disease in rats [27-29]. This model causes plaque accumulation around the ligature resulting in inflammatory cell recruitment and production of chemical mediators. Ultimately this leads to the destruction of periodontal hard and soft tissues in a manner similar to the progression of human periodontitis [30]. The structural, immunological, and microbiological properties of rat periodontal tissue are similar to that of human
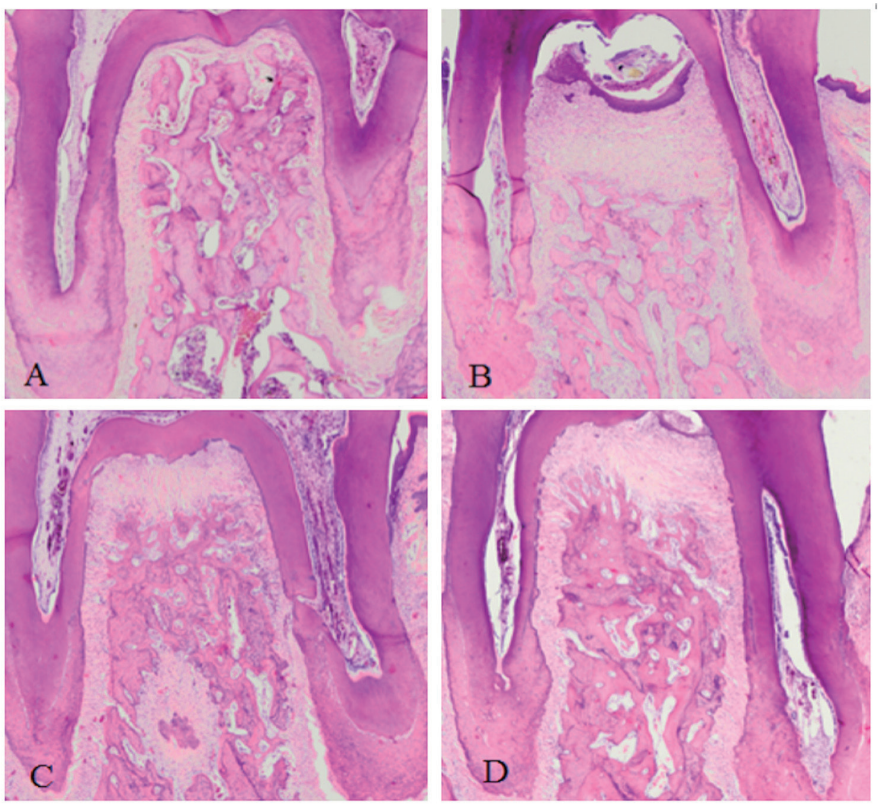

Fig. 4 Furcation area of the first molar tooth in the mesiodistal sections (magnification, 10×; bar= $100 \mu \mathrm{m})$. A, control group; B, periodontitis group; C, Pae group; D, saline group

Table 2 Levels of gingival VEGF

\begin{tabular}{cc}
\hline Groups & VEGF $(\mathrm{pg} / \mathrm{mL})^{\mathrm{a}}$ \\
\hline \multirow{2}{*}{1} & $11.56(10.25-12.56)$ \\
& $11.64 \pm 0.64$ \\
2 & $14.11(13.16-15.10)$ \\
3 & $14.18 \pm 0.62$ \\
& $19.98(18.96-20.57)$ \\
4 & $19.87 \pm 0.58$ \\
& $16.06(15.10-17.67)$ \\
\hline
\end{tabular}

$\overline{\text { Data are expressed as median, minimum, maximum, and mean } \pm \text { standard deviation. }}$ ${ }^{a}$ There are statistically significant differences between the groups $(P<0.008)$. Group 1 , control group; Group 2, periodontitis group; Group 3, Pae-administered group; Group 4, saline-administered group

molars [31-33].

Pae, a root extract of Paeonia lactiflora, is widely used in the treatment of chronic inflammatory diseases. Pae has been administered to rats for treatment with adjuvant arthritis $(25,50,100 \mathrm{mg} / \mathrm{kg} / \mathrm{day} ; 7$ days $)$, allergic contact dermatitis $(35,70,140 \mathrm{mg} / \mathrm{kg} /$ day; 7 days) and experimental periodontitis (30, $60 \mathrm{mg} / \mathrm{kg} / \mathrm{day} ; 7$ days) (Respectively; at various doses, lengths of time)[20,22,34]. The dose and duration of Pae $(100 \mathrm{mg} / \mathrm{kg} /$ day for 7 days) used in the present study was chosen based on studies evaluating the effects of Pae on inflammatory diseases. These studies noted that this dose has an anti-inflammatory effect without any side effects $[20,22,34]$. 
Given the historical lack of clinical and radiographic evaluation, histological analysis of block sections is essential to evaluate new therapeutic strategies, or when comparing therapeutic modalities [35]. In the present study, histomorphometric analysis was performed on block sections using reference points determined by anatomical constructions. Biochemically, the gingival VEGF levels were assessed by ELISA, a highly specific and sensitive method.

Based on histomorphometric analysis in the present study, the systemic application of Pae prevented alveolar bone resorption. The AA in the furcation area was significantly higher in the Pae group than in the saline group. Additionally, it appears that Pae has positive effects not only on alveolar bone but also on soft tissues, with the AL significantly reduced in the Pae group compared with the saline group. $\mathrm{Ni}$ et al. reported that alveolar bone resorption decreased significantly in Pae-administered groups [20]. Ni et al. also found that Pae decreased collagen fiber breakdown in a dosedependent manner, and that collagen fibers were more organized in the Pae-administered group than in the experimental periodontitis group in this study [20]. Similarly, studies with rheumatoid arthritis and collageninduced arthritis suggest that Pae reduces the amount of bone erosion and destruction [36,37].

The biochemical analyses in the present study found that VEGF levels increased significantly in the experimental periodontitis groups compared with the control group. Other studies have suggested that this angiogenic mediator has a regulatory effect on periodontal disease, but levels of VEGF have been found to increase [5,6,38], decrease [39], or remain unchanged [7]. VEGF has been shown to contribute to the spread of inflammation by increasing vascular permeability in studies noting increased VEGF levels in periodontal disease $[5,6]$. VEGF has also been found to be involved in the remodeling, repair, and regeneration of periodontal tissue [12].

According to the findings of the present experimental study, the highest levels of VEGF in the Pae group support studies suggesting that VEGF is more closely related to the healing stage of periodontal disease rather than to the tissue destruction stage [12]. The changes in gingival tissue VEGF levels support the histomorphometric findings in the present study, and Pae may be considered to be associated with periodontal tissue healing when this process and dose are used. Taken together, the results of the present study suggest the need for further studies.

\section{Acknowledgments}

This study was supported by the Scientific Research Fund of Ondokuz May1s University in Samsun/Turkey (Project number: PYO.DIS.1904.15.001).

\section{Conflict of interest}

The authors report no conflict of interest related to this study.

\section{References}

1. AlMoharib HS, AlMubarak A, AlRowis R, Geevarghese A, Preethanath RS, Anil SJ (2014) Oral fluid based biomarkers in periodontal disease: part1. Saliva. J Int Oral Health 6, 95-103.

2. Almerich-Silla JM, Montiel-Company JM, Pastor S, Serrano F, Puig-Silla M, Dasí F (2015) Oxidative stress parameters in saliva and its association with periodontal disease and types of bacteria. Dis Markers, doi: 10.1155/2015/653537.

3. Page RC, Kornman KS (1997) The pathogenesis of human periodontitis: an introduction. Periodontol 2000 14, 9-11.

4. Ferrara N (1996) Vascular endothelial growth factor. Eur J Cancer 32A, 2413-2422.

5. Johnson RB, Serio FG, Dai X (1999) Vascular endothelial growth factors and progression of periodontal diseases. J Periodontol 70, 848-852.

6. Suthin K, Matsushita K, Machigashira M, Tatsuyama S, Imamura T, Torii M et al. (2003) Enhanced expression of vascular endothelial growth factor by periodontal pathogens in gingival fibroblasts. J Periodontal Res 38, 90-96.

7. Keles GC, Cetinkaya BO, Eroglu C, Simsek SB, Kahraman H (2010) Vascular endothelial growth factor expression levels of gingiva in gingivitis and periodontitis patients with/ without diabetes mellitus. Inflamm Res 59, 543-549.

8. Kim KJ, Li B, Winer J, Armanini M, Gillett N, Phillips H et al. (1993) Inhibition of vascular endothelial growth factor-induced angiogenesis suppresses tumour growth in vivo. Nature 362, 841-844
9. Folkman J (1995) Angiogenesis in cancer, vascular, rheumatoid and other disease. Nat Med $1,27-31$.

10. Ferrara N, Davis-Smyth T (1997) The biology of vascular endothelial growth factor. Endocr Rev 18, 4-25.

11. Balli U, Keles GC, Cetinkaya BO, Mercan U, Ayas B, Erdogan D (2014) Assessment of vascular endothelial growth factor and matrix metalloproteinase-9 in the periodontium of rats treated with atorvastatin. J Periodontol 85, 178-187.

12. Cetinkaya BO, Keles GC, Ayas B, Sakallioglu EE, Acikgoz G (2007) The expression of vascular endothelial growth factor in a rat model at destruction and healing stages of periodontal disease. J Periodontol 78, 1129-1135.

13. Chang Y, Zhang L, Wang C, Jia XY, Wei W (2011) Paeoniflorin inhibits function of synoviocytes pretreated by rIL-1 $\alpha$ and regulates EP4 receptor expression. J Ethnopharmacol $137,1275-1282$

14. Zhang Y, Zhou R, Zhou F, Cheng H, Xia B (2014) Total glucosides of peony attenuates 2,4,6 trinitrobenzene sulfonic acid/ethanol-induced colitis in rats through adjustment of th1/th2 cytokines polarization. Cell Biochem Biophys 68, 83-95.

15. Chen M, Cao L, Luo Y, Feng X, Sun L, Wen M et al. (2015) Paeoniflorin protects against concanavalin A-induced hepatitis in mice. Int Immunopharmacol 24, 4249.

16. Wang Y, Dai J, Zhu Y, Zong W, Lu S, Chen H et al. (2018) Paeoniflorin regulates osteoclastogenesis and osteoblastogenesis via manipulating NF- $\mathrm{\kappa B}$ signaling pathway both in vitro and in vivo. Oncotarget 9, 7372 .

17. Wang Y, Zhu Y, Lu S, Hu C, Zhong W, Chai Y (2018) Beneficial effects of paeoniflorin on osteoporosis induced by high-carbohydrate, high-fat diet-associated hyperlipidemia in vivo. Biochem Biophys Res Commun 498, 981-987.

18. Li Z, Li D, Chen X (2018) Paeoniflorin inhibits receptor activator for nuclear factor $\kappa \mathrm{B}$ (RANK) ligand-induced osteoclast differentiation in vitro and particle-induced osteolysis in vivo. Med Sci Monit 24, 1044-1053.

19. He DY, Dai SM (2011) Anti-inflammatory and immunomodulatory effects of paeonia lactiflora pall., a traditional chinese herbal medicine. Front Pharmacol 2, 10.

20. Ni J, Yang D, Song L, Li C (2016) Protective effects of paeoniflorin on alveolar bone resorption and soft-tissue breakdown in experimental periodontitis. J Periodontal Res 51, 257-264.

21. Keles GC, Acikgoz G, Ayas B, Sakallıglu E, Firatli E (2005) Determination of systemically \& locally induced periodontal defects in rats. Indian J Med Res 121, 176-184.

22. Wu H, Wei W, Song L, Zhang L, Chen Y, Hu X (2007) Paeoniflorin induced immune tolerance of mesenteric lymph node lymphocytes via enhancing beta 2-adrenergic receptor desensitization in rats with adjuvant arthritis. Int Immunopharmacol 7, 662-673.

23. Balli U, Cetinkaya BO, Keles GC, Keles ZP, Guler S, Sogut MU et al. (2016) Assessment of MMP-1, MMP-8 and TIMP-2 in experimental periodontitis treated with kaempferol. J Periodontal Implant Sci 46, 84-95.

24. Kantarci A, Hasturk H, Van Dyke TE (2015) Animal models for periodontal regeneration and peri-implant responses. Periodontol 2000 68, 66-82.

25. Chang KM, Ryan ME, Golub LM, Ramamurthy NS, McNamara TF (1996) Local systemic factors in periodontal disease increase matrix-degrading enzyme activities in rat gingiva: effect of micocycline therapy. Res Commun Mol Pathol Pharmacol 91, 303-318.

26. Klausen B (1991) Microbiological and immunological aspects of experimental periodontal disease in rats: a review article. J Periodontol 62, 59-73.

27. Nassar CA, Nassar PO, Abi Rached RS, Holzhausen M, Marcantonio E Jr., Spolidorio LC (2004) Effect of cyclosporin A on alveolar bone homeostasis in a rat periodontitis model. J Periodontal Res 39, 143-148.

28. Gomes DA, Pires JR, Zuza EP, Muscara MN, Herrera BS, Spolidorio LC et al. (2009) Myeloperoxidase as inflammatory marker of periodontal disease: experimental study in rats. Immunol Invest 38, 117-122.

29. Herrera BS, Martins-Porto R, Maia-Dantas A, Campi P, Spolidorio LC, Costa SK et al. (2011) iNOS-derived nitric oxide stimulates osteoclast activity and alveolar bone loss in ligature-induced periodontitis in rats. J Periodontol 82, 1608-1615.

30. Guimaraes MR, Nassar PO, Andia DC, Nassar CA, Spolidorio DMP, Rossa C et al. (2007) Protective effects of tacrolimus, a calcineurin inhibitor, in experimental periodontitis in rats. Arch Oral Biol 52, 882-888.

31. Yamasaki A, Nikai H, Niitani K, Ijuhin N (1979) Ultrastructure of the junctional epithelium of germfree rat gingiva. J Periodontol 50, 641-648.

32. Weinberg MA, Bral M (1999) Laboratory animal models in periodontology. J Clin Periodontol 26, 335-340.

33. Struillou X, Boutigny H, Soueidan A, Layrolle P (2010) Experimental animal models in periodontology: a review. Open Dent J 4, 37-47.

34. Wang C, Yuan J, Wu HX, Chang Y, Wang QT, Wu YJ et al. (2013) Paeoniflorin inhibits inflammatory responses in mice with allergic contact dermatitis by regulating the balance between inflammatory and anti-inflammatory cytokines. Inflamm Res 62, 1035-1044.

35. Reddy MS, Jeffcoat MK (1999) Methods of assessing periodontal regeneration. Periodontol 2000 19, 87-103.

36. Zhu L, Wei W, Zheng Y-Q, Jia X-Y (2005) Effects and mechanisms of total glucosides of paeony on joint damage in rat collagen-induced arthritis. Inflamm Res 54, 211-220.

37. Wu D, Chen J, Zhu H, Xiong XG, Liang QH, Zhang Y et al. (2014) UPLC-PDA determination of paeoniflorin in rat plasma following the oral administration of radix paeoniae alba and its effects on rats with collagen-induced arthritis. Exp Ther Med 7, 209-217.

38. Oyama T, Sakuta T, Matsushita K, Maruyama I, Nagaoka S, Torii M (2000) Effects of roxithromycin on tumor necrosis factor-alpha-induced vascular endothelial growth factor expression in human periodontal ligament cells in culture. J Periodontol 71, 1546-1553.

39. Chapple CC, Kumar RK, Hunter N (2000) Vascular remodelling in chronic inflammatory periodontal disease. J Oral Pathol Med 29, 500-506. 